\title{
Arritmias cardíacas en habitantes de gran altura con diagnóstico de corazón pulmonar crónico
}

\author{
Cardiac arrhythmias in highlanders with a diagnosis of chronic cor pulmonale \\ Ricardo Sosa-Villarreal * y José M. Arce-Carreón ${ }^{2}$ \\ ${ }^{1}$ Departamento de Cardiología; ${ }^{2}$ Departamento de Electrofisiología. Instituto Nacional de Tórax. La Paz, Bolivia
}

\begin{abstract}
Resumen
Antecedentes: El corazón pulmonar crónico (CPC) muestra un incremento en habitantes que viven en grandes altitudes. Objetivo: Investigar la frecuencia de arritmias cardíacas y factores de riesgo para su desarrollo. Métodos: Estudio descriptivo y transversal; se revisó el registro de pacientes internados del Departamento de Cardiología del Instituto Nacional de Tórax, La Paz, Bolivia, entre enero de 2017 y junio de 2018; se incorporó a todos los individuos con diagnóstico de CPC, definido por criterios clínicos, electrocardiográficos y ecocardiográficos; se incluyó a 162 pacientes que cumplieron los criterios en el análisis; se utilizaron la t de Student y ANOVA. Resultados: Arritmias: fibrilación auricular (FA), 125 (75\%); aleteo auricular (AA), 17 (10.5\%); taquicardia auricular (TA), 17 (10.5\%); extrasístoles, 3 (1.9\%). Según el análisis univariado, los factores relacionados con el desarrollo de arritmias fueron: eritrocitosis: FA, RR: 1.33, otras arritmias (RR: 1.67), $p=0.0001$; hipertensión arterial pulmonar: $F A, R R: 3.10$, otras arritmias (RR: 3.21), $p=0.0001$; dilatación de aurícula derecha (AD): $F A$, RR: 1.92, otras arritmias (RR: 2.13), $p=0.0001$; obesidad: $F A, R R: 3.47, p=0.001$, otras arritmias (RR: 3.70$), p=0.001$; hipertensión arterial sistémica: FA, RR: 3.10, $p=0.001$, otras arritmias (RR: 3.21), $p=0.001$. Según el análisis multivariado: eritrocitosis (RR: 2.2), dilatación de $A D$ (RR: 1.2), $p=0.0001$. Conclusiones: Se encontró FA con mayor frecuencia en los pacientes con CPC; los factores de riesgo con mayor significancia estadística para su presentación fueron la eritrocitosis y la dilatación de la $A D$.
\end{abstract}

Palabras clave: Fibrilación auricular. Hipertensión arterial pulmonar. Eritrocitosis.

\begin{abstract}
Background: Chronic cor pulmonale (CPC), with increased presentation in high-altitude inhabitants. Objectives: Investigating the frequency of cardiac arrhythmias, and risk factors for its development. Methods: Descriptive, cross-sectional study, the inpatient registry of the Department of Cardiology of the National Institute of Torax, La Paz-Bolivia, from January 2017 to June 2018 was reviewed, all were incorporated with the diagnosis of CPC, defined by clinical criteria, electrocardiographic and echocardiographic, 162 patients who met the criteria were taken, the student's t-test and ANOVA were used for the analysis. Results: Arrhythmias: atrial fibrillation (AF) 125 (75\%), atrial flutter (AA) 17 (10.5\%), atrial tachycardia (AT) 17 (10.5\%),
\end{abstract}

\section{Correspondencia:}

*Ricardo Sosa-Villarreal

Departamento de Cardiología

Instituto Nacional de Tórax

Fecha de recepción: 08-11-2019

Fecha de aceptación: 22-03-2020

E-mail: costarestera@ hotmail.com

DOI: 10.24875/ACM.20000367
Disponible en internet: 06-10-2020 Arch Cardiol Mex. 2020;90(4):415-419 www.archivoscardiologia.com

1405-9940 / () 2020 Instituto Nacional de Cardiología Ignacio Chávez. Publicado por Permanyer. Este es un artículo open access bajo la licencia CC BY-NC-ND (http://creativecommons.org/licenses/by-nc-nd/4.0/). 
extrasystoles $3(1.9 \%)$. Univariate analysis of factors related to the development of arrhythmias: erythrocytosis: $F A, R R: 1.33$, other arrhythmias RR: 1.67, $p=0.0001$, pulmonary arterial hypertension: $F A, R R: 3.10$, other arrhythmias $R R: 3.21, p=$ 0.0001, right atrial dilation $(A D): F A, R R: 1.92$, other arrhythmias $R R: 2.13, p=0.0001$, obesity: $F A, R R: 3.47, p=0.001$, other arrhythmias RR: 3.70, $p=0.001$, systemic arterial hypertension: FA: RR: 3.10, $p=0.001$, other arrhythmias RR: 3.21, $p=0.001$. Multivariate analysis: erythrocytosis (RR: 2.2), $A D$ dilation (RR: 1.2), $p=0.0001$. Conclusions: $A F$ was found more frequently in patients with $C P C$, the risk factors with the greatest statistical significance for presentation were: erythrocytosis and $A D$ dilation.

Key words: Atrial fibrillation. Pulmonary arterial hypertension. Erythrocytosis.

\section{Introducción}

Las ciudades de La Paz y El Alto se encuentran a 3,577 y 4,050 msnm $^{1}$ respectivamente y en ambas reside una población de 2,904,996 habitantes, de los cuales la mitad se halla repartida en distintos pisos ecológicos del departamento de La Paz; la presión barométrica a esta altitud es de $490 \mathrm{mmHg}$ y la $\mathrm{FIO}_{2}$ de $0.21(21 \%)$, con variaciones climáticas muy especiales y temperaturas frías y secas.

Como mecanismo de aclimatación crónica, los nativos de esta altura tienen valores de presión pulmonar mayores respecto de quienes viven a nivel del mar, con presión pulmonar normal para la altura ${ }^{1}$.

El corazón pulmonar crónico (CPC) es la alteración estructural (hipertrofia o dilatación) y el deterioro funcional del ventrículo derecho (VD) resultados de la elevación de las presiones pulmonares en relación con enfermedades del parénquima pulmonar, su vascularización, la vía respiratoria superior o la pared torácica. La disfunción del VD debida a enfermedades cardíacas del lado izquierdo o cardiopatías congénitas no se considera $\mathrm{CPC}^{2}$.

En el ámbito de trabajo en que se encuentra esta población, la CPC es una cardiopatía frecuente ${ }^{3,4}$, efecto de alteraciones que cursan con hipoxemia $u$ obstrucción arterial vascular pulmonar. Tiene un sólido nexo con enfermedad pulmonar obstructiva crónica, secuelas crónicas de tuberculosis pulmonar y enfermedad tromboembólica pulmonar.

La incidencia y prevalencia del CPC es variable debido a la diversidad de entidades que lo desencadenan y, en consecuencia, su epidemiología está ligada a las causas que lo originan.

\section{Material y métodos}

Este estudio es de tipo descriptivo y transversal, y se elaboró en el período de enero de 2017 a junio de 2018. Los objetivos fueron determinar cuáles eran las arritmias cardíacas más frecuentes en pacientes con diagnóstico de CPC internados en el Departamento de Cardiología del Instituto Nacional de Tórax, La Paz, Bolivia, y conocer los factores de riesgo predisponentes para el desarrollo de arritmias cardíacas en éstos. Para la recolección de datos se consultó el registro clínico de los pacientes internados en este hospital; los criterios de inclusión fueron los pacientes con diagnóstico establecido de CPC a través de criterios clínicos (segundo ruido cardíaco aumentado, presencia de soplo de regurgitación tricuspídeo o de insuficiencia pulmonar, congestión venosa sistémica), electrocardiográficos (índices de Sokolow-Lyon derecho > $11 \mathrm{~mm}$, y Lewis < -14 mm) y ecocardiográficos, con las dimensiones del VD y la determinación de la presión arterial pulmonar sistólica (PAPS) obtenidas por este mismo método (incremento del espesor de la pared libre del VD > $5 \mathrm{~mm}$, dilatación del segmento basal del VD > $42 \mathrm{~mm}$, longitud del VD > $86 \mathrm{~mm}$, insuficiencia tricuspídea, PAPS > $35 \mathrm{mmHg}$, dilatación de la aurícula derecha $\left.(A D)>18 \mathrm{~cm}^{2}\right)$, con residencia permanente en las ciudades de La Paz y El Alto, sin incluir a los pacientes con enfermedades distintas de la hipertensión pulmonar (cardiopatía hipertensiva, cardiopatía isquémica, cardiopatía reumática).

Los factores de riesgo encontrados fueron eritrocitosis patológica de la altura (hemoglobina $>17 \mathrm{~g} / \mathrm{dl}$ para mujeres y $>18 \mathrm{~g} / \mathrm{dl}$ para varones y un hematócrito > $62.1 \%)^{5}$, dislipidemia (colesterol total $>200 \mathrm{mg} / \mathrm{dl}, \mathrm{HDL}$ $<70 \mathrm{mg} / \mathrm{dl})^{6}$, hipertensión arterial sistémica (presión arterial sistólica > $130 \mathrm{mmHg}$ y presión diastólica > $80 \mathrm{mmHg})^{7}$, diabetes mellitus tipo 2 establecida y controlada $\left(\mathrm{HbA}_{1 \mathrm{c}}<7.0 \%\right)^{8}$, obesidad $\left(\mathrm{IMC}>25 \mathrm{~kg} / \mathrm{m}^{2}\right)^{8} \mathrm{y}$ tabaquismo.

Se identificaron arritmias supraventriculares como la fibrilación auricular ( $\mathrm{FA})$, aleteo auricular $(\mathrm{AA})$, taquicardia auricular (TA), extrasístoles ventriculares y supraventriculares; no se identificaron arritmias ventriculares. Como datos del ECG de las características de la $\mathrm{FA}$, la frecuencia promedio de $78 \mathrm{lpm}$ y la presencia de bajo voltaje en el plano frontal probablemente se atribuyeron al sobrepeso o afección pulmonar y 
Tabla 1. Características generales de los pacientes

\begin{tabular}{|c|c|}
\hline & Global (162) \\
\hline Edad & $68.7 \pm 12$ \\
\hline $\begin{array}{l}\text { Género } \\
\text { Masculino } \\
\text { Femenino }\end{array}$ & $\begin{array}{l}77(47.5 \%) \\
85(52.5 \%)\end{array}$ \\
\hline $\begin{array}{l}\text { Hipertensión arterial pulmonar } \\
\text { Hipertensión arterial pulmonar leve (PAP, } \\
35-50 \mathrm{mmHg} \text { ) } \\
\text { Hipertensión arterial pulmonar moderada a } \\
\text { grave (PAP, } 51-70 \mathrm{mmHg} \text { ) }\end{array}$ & $\begin{array}{l}27(16.7 \%) \\
135(83.3 \%)\end{array}$ \\
\hline Eritrocitosis & $95(56.8 \%)$ \\
\hline Tabaquismo & $41(25.3 \%)$ \\
\hline Obesidad & $34(24 \%)$ \\
\hline Hipertensión arterial sistémica & $63(38.9 \%)$ \\
\hline Dislipidemia & $56(34.6 \%)$ \\
\hline Diabetes mellitus & $8(4.9 \%)$ \\
\hline $\begin{array}{l}\text { Dilatación de la aurícula derecha } \\
\left.\text { Dilatación leve (área }<18 \mathrm{~cm}^{2}\right) \\
\text { Dilatación moderada a grave (área, 18-26 cm²) } \\
\text { Dilatación del ventrículo derecho }\end{array}$ & $\begin{array}{l}27(16.7 \%) \\
135(83.3 \%) \\
162(100 \%)\end{array}$ \\
\hline
\end{tabular}

bloqueo de rama derecha en los pacientes de 70 años, sin encontrar bloqueo de rama izquierda.

Para el análisis se empleó el paquete estadístico SPSS versión 23.0 y se obtuvieron las frecuencias absolutas, las frecuencias relativas y las tablas de contingencia; para el análisis de los factores relacionados se realizó la prueba t de Student para el análisis univariado y la prueba ANOVA para el multivariado; en uno y otro análisis se calculó el riesgo relativo (RR) de los factores relacionados.

\section{Resultados}

Se analizó a un total de 224 pacientes; 62 no cumplieron los criterios de inclusión del estudio y 162 satisficieron los criterios de inclusión clínicos, electrocardiográficos y ecocardiográficos, con una edad promedio de $68.7 \pm 12$ años (52.5\% femenino); en la tabla 1 se describen las características generales.

Se reconoció que las arritmias más frecuentes eran la FA con 125 (77\%), el AA con $17(11 \%)$, la TA con $17(10 \%)$, y las extrasístoles ventriculares y supraventriculares con $3(2 \%)$; no se identificaron episodios de taquicardia ventricular (Fig. 1).

En el análisis univariado (Tabla 2), los principales factores relacionados para el desarrollo de arritmias fueron
Tabla 2. Análisis univariado

\begin{tabular}{|c|c|c|c|}
\hline & $\begin{array}{l}\text { FA y otras } \\
\text { arritmias } \\
(n=162)\end{array}$ & $\begin{array}{l}\text { Riesgo } \\
\text { relativo } \\
\text { (IC95\%) }\end{array}$ & $\begin{array}{l}\text { Valor } \\
\text { de } p\end{array}$ \\
\hline $\begin{array}{l}\text { Eritrocitosis } \\
\text { FA } \\
\text { Otras arritmias }\end{array}$ & $\begin{array}{l}83(87.4 \%) \\
12(12.6 \%)\end{array}$ & $\begin{array}{l}1.33 \\
1.67\end{array}$ & $\begin{array}{l}0.0001 \\
0.0001\end{array}$ \\
\hline $\begin{array}{l}\text { Hipertensión arterial } \\
\text { pulmonar } \\
\text { FA } \\
\text { Otras arritmias }\end{array}$ & $\begin{array}{c}125(77.2 \%) \\
37(22.8 \%)\end{array}$ & $\begin{array}{l}3.10 \\
3.21\end{array}$ & $\begin{array}{l}0.0001 \\
0.0001\end{array}$ \\
\hline $\begin{array}{l}\text { Dilatación moderada a } \\
\text { grave de la aurícula } \\
\text { derecha } \\
\text { FA } \\
\text { Otras arritmias }\end{array}$ & $\begin{array}{l}94(75.2 \%) \\
30(18.5 \%)\end{array}$ & $\begin{array}{l}1.92 \\
2.13\end{array}$ & $\begin{array}{l}0.0001 \\
0.0001\end{array}$ \\
\hline $\begin{array}{l}\text { Obesidad } \\
\text { FA } \\
\text { Otras arritmias }\end{array}$ & $\begin{array}{c}30(18.5 \%) \\
4(2.46 \%)\end{array}$ & $\begin{array}{l}3.47 \\
3.70\end{array}$ & $\begin{array}{l}0.001 \\
0.001\end{array}$ \\
\hline $\begin{array}{l}\text { Hipertensión arterial } \\
\text { sistémica } \\
\text { FA } \\
\text { Otras arritmias }\end{array}$ & $\begin{array}{c}50(30.8 \%) \\
13(8 \%)\end{array}$ & $\begin{array}{l}3.10 \\
3.21\end{array}$ & $\begin{array}{l}0.001 \\
0.001\end{array}$ \\
\hline
\end{tabular}

FA: fibrilación auricular.

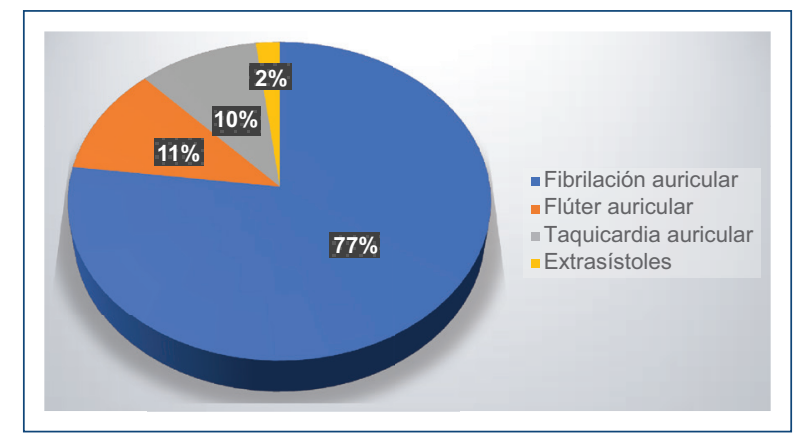

Figura 1. Frecuencia de arritmias.

eritrocitosis, hipertensión arterial pulmonar moderada, dilatación moderada y grave de la $A D$, obesidad e hipertensión arterial sistémica. Al realizar el análisis multivariado (Tabla 3) se observó una mayor significancia estadística con la eritrocitosis y la dilatación moderada a grave de $A D$ como factores promotores del desarrollo de arritmias supraventriculares, en particular la FA.

\section{Discusión}

Las ciudades de La Paz y El Alto (Bolivia) tienen una población nativa cuantiosa de gran altura, lo cual da 
Tabla 3. Análisis multivariado de los factores de riesgo (ANOVA) para el desarrollo de arritmias

\begin{tabular}{|l|c|c|}
\hline Factores de riesgo & $\begin{array}{c}\text { Riesgo relativo } \\
\text { (RR) }\end{array}$ & $\mathbf{P}$ \\
\hline Eritrocitosis & 2.2 & 0.0001 \\
\hline Dilatación de la aurícula derecha & 1.2 & 0.0001 \\
\hline Hipertensión arterial pulmonar & 1.01 & 0.001 \\
\hline
\end{tabular}

lugar a que los pacientes con arritmias en esta población sean de especial interés; algunos de estos aspectos fisiopatológicos que intervienen en su desarrollo difieren del resto de las poblaciones que viven a una menor altura ${ }^{1}$.

Los nativos que residen a gran altura tienen en realidad una presión pulmonar mayor respecto de quienes viven a nivel del mar'; esto, sumado a la gran incidencia de afección pulmonar y tuberculosis presentes en este medio, contribuye a que la incidencia de CPC sea muy elevada ${ }^{3}$.

Se ha advertido además que, por diversos factores, los pacientes con CPC tienden a desarrollar arritmias cardíacas, sobre todo supraventriculares ${ }^{9}$, entre las cuales figura la dilatación de la $A D^{10}$, secundaria a la grave hipertensión pulmonar relacionada con esta enfermedad, y ambas desencadenan una alteración en la remodelación auricular ${ }^{11}$.

Muy pocos estudios en el plano mundial describen la relación con estos aspectos; la mayoría corresponde a descripciones de informes aislados sobre la incidencia de arritmias cardíacas a gran altura.

Cabe esperar que en la dilatación de la $A D$ vinculada con hipoxia crónica, las arritmias cardíacas reconocidas más a menudo sean el $A A$, las extrasístoles supraventriculares y la TA; sin embargo, en este protocolo se observó que la principal arritmia cardíaca en pacientes con CPC era la FA en un gran porcentaje (77\%) y, con mucha menor frecuencia, el AA (11\%); esto supone que, además de otros factores distintos de la hipertensión pulmonar y la dilatación de las cavidades derechas secundaria a la hipoxemia crónica, puede intervenir la génesis de las arritmias supraventriculares, ente ellas la eritrocitosis.

En el análisis multivariado se encontró como factor de riesgo más importante para el desarrollo de FA la presencia de eritrocitosis (RR, 2.02; $p=0.0001) \mathrm{y}$, en menor medida, la dilatación moderada de la aurícula derecha (RR, 1.02).

Una de las causas de hipertensión pulmonar es el mal de montaña crónico (que se define como la pérdida de adaptación a grandes altitudes), que puede ser primario o efecto de enfermedad pulmonar, obesidad, apnea del sueño y edad avanzada. Como consecuencia, se activa una respuesta de compensación y aumentan la secreción de eritropoyetina y la carga eritrocitaria $^{12}$; la presencia de hipertensión pulmonar secundaria incrementa la mortalidad de tal modo que estos casos se relacionan con gran hipertrofia VD, excesiva muscularización de las arterias pulmonares distales y engrosamiento de la adventicia ${ }^{13}$.

Se ha postulado que la hipoxia crónica a grandes alturas, sumada a la pérdida de aclimatación a la altitud de forma crónica (mal de montaña crónico) e inflamación crónica, podría propiciar el establecimiento de un sustrato fisiopatológico, conocido como triple hipoxia, e influir en el desarrollo acelerado de la eritrocitosis patológica de altura ${ }^{13}$.

El desarrollo de eritrocitosis secundaria en un paciente portador de CPC que habita en grandes alturas es por tanto un factor pronóstico adverso que predispone al desarrollo de FA y ésta a su vez contribuye al deterioro hemodinámico de estos pacientes ${ }^{9,11}$.

Se ha observado además que la probabilidad de desarrollar episodios cardiovasculares en los próximos 10 años es mayor en sujetos con eritrocitosis, en comparación con pacientes sin ella, y que esta probabilidad se eleva en el subgrupo de individuos con hipertensión arterial pulmonar moderada a grave ${ }^{14}$.

Una hipótesis que pueda explicar la fisiopatología de la FA señala que de forma inicial se produce la hipoxia de grandes alturas y, en algunos casos, ésta puede sumarse a la pérdida crónica de aclimatación, lo que provoca hipoventilación alveolar crónica ${ }^{15}$ y a continuación estimulación mayor de la producción de eritropoyetina ${ }^{16}$, lo que aumenta la producción de glóbulos rojos.

Una vez establecida la eritrocitosis patológica se produce un estado inflamatorio crónico y alteración de la reología vascular por mayor viscosidad sanguínea, que a su vez predispone a la hipertensión arterial ${ }^{17}$ y lleva asimismo a una disminución de la oxigenación tisular, con aparición de disfunción endotelial.

La enfermedad cardíaca crónica, el estado inflamatorio constante y la disfunción endotelial son sustratos descritos de manera característica para la génesis de la $\mathrm{FA}^{18,19}$, al igual que la hipertensión arterial ${ }^{20}$, pero no la eritrocitosis como un factor predisponente.

La inflamación causa en parte la remodelación estructural y eléctrica de la aurícula; en presencia de ésta se acentúa la heterogeneidad de la conducción auricular al alterar la expresión de las conexinas, un factor que altera el acoplamiento intercelular ${ }^{21}$. 
Una vez que se establece la FA de forma crónica empeora el estado clínico del enfermo con $\mathrm{CPC}^{9}$ y aumenta a su vez el deterioro hemodinámico del paciente. Por esta razón, el desarrollo de eritrocitosis se convierte en un factor de riesgo destacable en la génesis de la FA y por ende de mal pronóstico en pacientes portadores de CPC.

\section{Conclusiones}

La arritmia más frecuente en habitantes de gran altura portadores de CPC es la FA. Los factores de riesgo para el desarrollo de arritmias cardíacas, sobre todo la FA en pacientes con CPC que viven a gran altitud, son la eritrocitosis y la dilatación grave de la AD.

\section{Agradecimientos}

Los autores expresan su agradecimiento al personal médico del Instituto Nacional de Tórax.

\section{Financiamiento}

La presente investigación no recibió ayuda económica proveniente de agencias del sector público, privado o comercial.

\section{Conflicto de intereses}

Ninguno.

\section{Responsabilidades éticas}

Protección de personas y animales. Los autores declaran que para esta investigación no se han realizado experimentos en seres humanos ni en animales.

Confidencialidad de los datos. Los autores declaran que han seguido los protocolos de su centro de trabajo sobre la publicación de datos de pacientes.

Derecho a la privacidad y consentimiento informado. Los autores han obtenido el consentimiento informado de los pacientes o sujetos referidos en el artículo. Este documento obra en poder del autor de correspondencia.

\section{Bibliografía}

1. Instituto Boliviano de Biología de Altura (IBBA), Comité Organizador de los VIII Juegos Deportivos Bolivarianos. Parámetros Biológicos Normales. 1ra ed. La Paz-Bolivia, 1977.

2. Budev MM, Arroliga AC, Wiedemann HP, Matthay RA. Cor pulmonale: an overview. Semin Respir Crit Care Med. 2003;24(3):233-43.

3. Díaz LA. Cor pulmonale crónico: aspectos clínicos epidemiológicos en adultos de altura. Rev Per Cardiol. 2009;35(1):44-52.

4. 2bago.bo [Internet]. La Paz: Cor pulmonale crónico: causas, enfermedades asociadas y características evolutivas en 2172 pacientes internados, Hospital Obrero N 1, La Paz, Bolivia; C2017 (consultado 18 febrero de 2017). Disponible en: https://www2.bago.com.bo/sbc/latido/Vol5_n6/html/ cor_pulmonale.htm

5. Amaru R, Torres G, Quispe T, Mamani J, Peñaloza R, Miguez H. Eritrocitosis patológica de la altura. 1ra ed. La Paz: Unidad de Biología Italo-Boliviano, 2016

6. Catapano AL, Graham I, Backer G, Wiklund O, Chapman MJ, Drexhel H, et al. Guía ESC/EAS 2016 sobre el tratamiento de las dislipemias, Grupo de Trabajo de la Sociedad Europea de Cardiología (ESC) y la European Atherosclerosis Society (EAS) sobre el tratamiento de las dislipemias desarrollada con la colaboración especial de la European Association for Cardiovascular Prevention \& Rehabilitation (EACPR). Rev Esp Cardiol. 2017;70(2):115.e1-e64.

7. Reboussin DM, Allen NB, Griswold ME, Guallar E, Hong Y, Lackland DT, et al. Systematic Review for the 2017 ACC/AHA/AAPA/ABC/ACPM/AGS/ APhA/ASH/ASPC/NMA/PCNA Guideline for the Prevention, Detection, Evaluation, and Management of High Blood Pressure in Adults: A Report of the American College of Cardiology/American Heart Association Task Force on Clinical Practice Guidelines. Hypertension 2018;71(6):e116-e135.

8. Piepoli MF, Hoes AW, Agewall S, Albus C, Brotons C, Catapano AL, et al. Guía ESC 2016 sobre prevención de la enfermedad cardiovascular en la práctica clínica, Sexto Grupo de Trabajo Conjunto de la Sociedad Europea de Cardiología y otras Sociedades sobre Prevención de Enfermedad Cardiovascular en la Práctica Clínica (constituido por representantes de 10 sociedades y expertos invitados) desarrollada con la contribución especial de la European Association for Cardiovascular Prevention \& Rehabilitation (EACPR). Rev Esp Cardiol. 2016;69(10):939.e1-e87.

9. Hernández SP, Lázaro SM, Alcalá JE, Macías CB. Cor pulmonale. Medicine 2017:12(35):2116-26.

10. Waligóra M, Tyrka A, Miszalski TJ, Urbanczyk ZM, Podolec P, Kopéc G, et al. Right atrium enlargement predicts clinically significant supraventricular arrhythmia in patients with pulmonary arterial hypertension. Heart and Lung 2018;47(3):237-242.

11. Smith B, Genuardi MV, Koczo A, Zou RH, Thoma FW, Handen A, et al. Atrial arrhythmias are associated with increased mortality in pulmonary arterial hypertension. Pulm Circ. 2018;8(3):1-9.

12. Peñaloza $D$. Efectos de la exposición a grandes alturas en la circulación pulmonar. Rev Esp Cardiol. 2012;65(12):1075-78.

13. Peñaloza D, Arias SJ. The heart and pulmonary circulation at high altitude. Healthy highlanders and chronic mountain sickness. Circulation 2007; 115(9):1132-46.

14. Prchal JT, Prchal JF. Williams hematology. $9^{\text {th }}$ ed. New York: McGraw Hill, 2015.

15. Acinelli RA, López LM. Enfermedades por exposición a la altura. Arch Bronconeumol. 2018;54(3):115-116.

16. Uscamayta QN. Eritrocitosis de altura patológica. Rev Cientif. 2007:5:50-6.

17. Marzal $D$, Rodríguez L. Etiología y prevención de la fibrilación auricular. Rev Esp Cardiol Supl. 2016;16(A):8-11.

18. Ramírez JD, Agudelo FJ, Correa R, Gonzales E. Fisiopatología de la fibrilación auricular. Rev Colomb Cardiol. 2016;23(S5):9-14.

19. Patel $P$, Dokainish $H$, Tsai $P$, Lakkis $N$. Update on the association of inflammation and atrial fibrillation. J Cardiovasc Electrophysiol. 2010;21(9):1064-70.

20. Aldashev AA, Sarybaev AS, Sydykov AS, Kalmyrzaev BB, Kim EV, Mamanova LB, et al. Characterization of high-altitude pulmonary hypertension in the kyrgyz: association with angiotensin-converting enzyme genotype. Am J Respir Crit Care Med. 2002;166(10):1396-1402.

21. Corante C, Ramírez CA, Figueroa R, Macarlupu JL, Galindo GV, Bilo G, et al. Excessive erythrocytosis and cardiovascular risk in andean highlanders. High Alt Med Biol. 2018;19(3):221-231. 